\title{
Correlation between erythrocyte aldose reductase level and human diabetic retinopathy
}

\author{
N Oishi, E Kubo, Y Takamura, K Maekawa, T Tanimoto, Y Akagi
}

Br J Ophthalmol 2002;86:1363-1366

Aim: To examine the relation between aldose reductase (AR) and the development and progression of diabetic retinopathy by comparing the erythrocyte AR levels with the prevalence of diabetic retinopathy in NIDDM patients. Methods: A clinic based cross sectional study was used. 611 NIDDM patients and 73 controls were enrolled. Erythrocyte AR levels were determined by ELISA. These AR levels were then correlated with patient age, duration of diabetes, and $\mathrm{HbA}_{\mathrm{lc}}$ levels. AR levels were also correlated with the prevalence of diabetic retinopathy in the entire NIDDM patient group and in three subgroups formed by separating the NIDDM patients by their duration of diabetes. The prevalence of diabetic retinopathy significantly increased with increased erythrocyte AR levels in patients with duration of diabetes of less than 10 years. A similar, but non-significant correlation between the prevalence of retinopathy and increased erythrocyte AR levels was observed in patients with diabetes duration of $10-20$ and $\geqslant 20$ years.

Results: The prevalence of diabetic retinopathy increased with increased erythrocyte AR levels in NIDDM patients with a duration of diabetes of less than 10 years.

Conclusion: It was suggested that the inhibition of $A R$ in patients with early NIDDM might be beneficial in reducing the development of diabetic retinopathy.

$\mathrm{D}$ iabetic eye complications include corneal epitheliopathy, cataract, and retinopathy. Of these, retinopathy is the most severe complication because it can result in irreversible blindness. While visual prognoses of patients with diabetic retinopathy may often be improved by photocoagulation or vitreous surgery, blindness cannot be prevented in all cases. Therefore, elucidating the mechanism for initiating diabetic retinopathy is important so that specific methods for the prevention and/or treatment of diabetic retinopathy can be developed. It is well established that high blood glucose levels greatly influence the development and progression of diabetic retinopathy. ${ }^{12}$ However, the involvement of other factors cannot be ruled out. Retinal capillary pericytes contain the enzyme aldose reductase (AR) and the accumulation of excess sugar alcohol, catalysed by AR in pericytes, has been linked to their degeneration and selective death. ${ }^{34}$ Pericyte degeneration, which is considered the hallmark of early diabetic retinopathy, also occurs in diabetic and galactose fed dogs that develop retinopathy. In these dogs pericyte degeneration has been prevented in a dose dependent manner with aldose reductase inhibitors. ${ }^{5-7}$ Mounting evidence for an involvement of AR as a risk factor for diabetic complications comes from studies of gene polymorphism. These studies suggest the presence of a putative "high risk" allele, Z-2 and a putative "protective" allele, $\mathrm{Z}+2$ that appear to have a role in modulating the expression level of AR. ${ }^{8}$ Several reports indicate that the Z-2 allele of AR is significantly higher in diabetics with retinopathy ${ }^{90}$ while the $\mathrm{Z}+2$ allele of $A R$ is associated with a lower rate of retinopathy. In addition, the $\mathrm{Z}-2$ allele appears to be associated with higher erythrocyte AR levels. ${ }^{11}$ Since erythrocyte AR levels can easily be determined in a clinical setting, we have investigated whether a correlation between human diabetic retinopathy and increased erythrocyte AR levels exists in 611 NIDDM patients treated at the Fukui Medical University.

\section{PATIENTS AND METHODS}

A clinic based cross sectional study was used. Subjects consisted of 611 patients with non-insulin dependent diabetes mellitus (NIDDM) who were consecutively treated from 1996 until 1998 at the Fukui Medical University and 73 nondiabetic patients. All consented to participate in this clinical trial. NIDDM was based on the diagnostic criteria of the Japan Diabetes Society. The fundus of each patient was evaluated by both direct ophthalmoscopy and colour photography and diabetic retinopathy was defined as the presence of retinal microaneurysm, dot haemorrhages, and/or exudates according to the diagnostic criteria of the Japan Society of Ophthalmic Diabetology.

After receiving informed consent, blood was drawn from each patient and erythrocytes were separated from $1 \mathrm{ml}$ of heparinised whole blood. Following the addition of an equal volume of $0.02 \mathrm{M}$ phosphate buffer ( $\mathrm{pH} 7.0$ ), the erythrocytes were haemolysed by two cycles of freezing and thawing. This was followed by centrifugation at $15000 \mathrm{~g}$ for 10 minutes. Erythrocyte AR levels were determined by ELISA using antihuman AR monoclonal antibody. Samples containing AR were initially placed in microplate wells containing solidified rabbit-antihuman AR polyclonal antibody, and allowed to react with the solidified anti-AR antibody (primary reaction). Mouse-antihuman AR monoclonal antibody labelled with horseradish peroxidase (enzyme labelled antibody) was then added to the reactive solution, and the AR enzyme previously bound to solidified AR antibody was allowed to bind with the enzyme labelled antibody (secondary reaction). Depending on the levels of AR present, a triple complex consisting of solidified antibody-AR-enzyme labelled antibody was also formed. Hydrogen peroxide and $o$-phenylenediamine were then added to each microplate well and the $o$-phenylenediamine substrate was oxidised by the peroxidase bound on the mouseantihuman AR monoclonal antibody. AR levels bound to the mouse-antihuman AR monoclonal antibody and bound in a triple complex were determined by spectrophotometric measurement of peroxidase activity at $490 \mathrm{~nm}$.

Correlations between the duration of diabetes in the overall NIDDM group and the prevalence of diabetic retinopathy were investigated along with comparisons of erythrocyte AR levels with age, duration of diabetes, and $\mathrm{HbA}_{\mathrm{lc}}$ levels. Erythrocyte AR levels were compared to the prevalence of retinopathy in the entire NIDDM group, and in three subgroups formed by dividing the NIDDM group according to duration of diabetes ( $<10$ years, 11-20 years, and $>20$ years) using the $\chi^{2}$ test. 


\begin{tabular}{lll}
$\begin{array}{l}\text { Table } 1 \\
\text { controls }\end{array}$ & Clinical features of NIDDM patients and \\
\hline & NIDDM & Control \\
\hline Number & 611 & 73 \\
(Male/female) & $(324 / 287)$ & $(30 / 43)$ \\
Age (years) & $60.8(11.4)$ & $65.5(11.3)$ \\
Duration (years) & $11.6(8.6)$ & - \\
$\mathrm{FBG}(\mathrm{mg} / \mathrm{dl})$ & $172(55)$ & $100(16)$ \\
$\mathrm{HbA}(\%)$ & $8.1(1.8)$ & - \\
Aldose reductase $(\mathrm{ng} / \mathrm{mg} \mathrm{Hb})$ & $10.5(2.9)$ & $10.1(3.4)$ \\
\hline \multicolumn{3}{l}{ Values are mean (SD). } \\
\end{tabular}



Figure 1 Relation between erythrocyte aldose reductase levels and age of NIDDM patients ( $n=611)$. Overall, a slight but significant decrease in erythrocyte AR levels was observed with age $(p=0.037)$. $r=$ correlation coefficients.

\section{RESULTS}

Studies were conducted on 611 NIDDM and 73 non-diabetic patients. The mean duration of diabetes and the mean ages, fasting blood glucose levels and $\mathrm{HbA}^{\text {Ic }}$ levels for each group are summarised in Table 1. Erythrocyte AR levels were determined for each participant by ELISA. Comparison of erythrocyte AR levels indicated that the mean level in NIDDM patients (10.5 (SD 2.9) $\mathrm{ng} / \mathrm{mg} \mathrm{Hb}$ ) was similar to that in controls (10.1 (3.4) $\mathrm{ng} / \mathrm{mg} \mathrm{Hb}$ ). Comparisons between the erythrocyte AR levels in all NIDDM patients and their age, duration of diabetes, and $\mathrm{HbA}_{\mathrm{lc}}$ levels are summarised in Figures 1-3. For the entire NIDDM population examined $(n=611)$, a slight but significant decrease in erythrocyte AR levels was observed with age $(\mathrm{p}=0.037)$ as well as duration of diabetes $(\mathrm{p}=0.032)$ (Figs 1 and 2). However, no significant correlation between erythrocyte AR levels and $\mathrm{HbA}_{\mathrm{lc}}$ levels $(\mathrm{p}=0.736)$ was observed (Fig $3)$. The level of $\mathrm{HbA}_{\mathrm{Ic}}$ was significantly decreased with the age of diabetic patients (Fig 4).

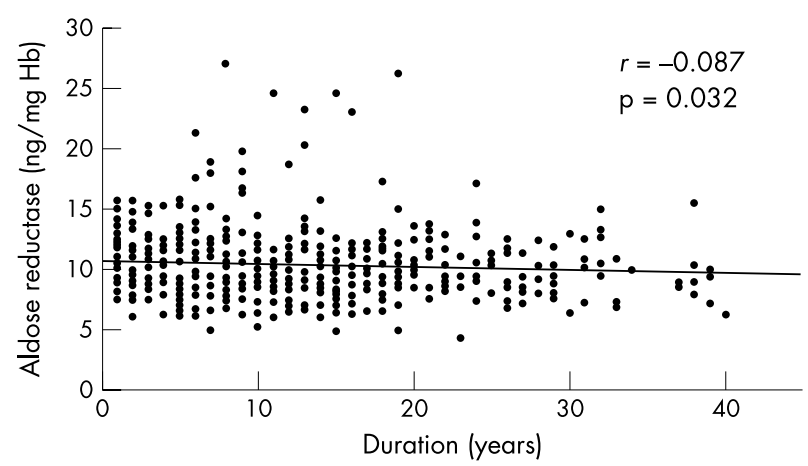

Figure 2 Relation between erythrocyte aldose reductase levels and duration of diabetes in NIDDM patients $(n=611)$. Overall, a slight but significant decrease in erythrocyte AR levels was observed with duration of diabetes $(p=0.032) . r=$ correlation coefficients.



Figure 3 Relation between erythrocyte aldose reductase levels and haemoglobin $A_{1 c}$ in diabetic patients $(n=611)$. No significant correlation is evident. $(p=0.736) . r=$ correlation coefficients.

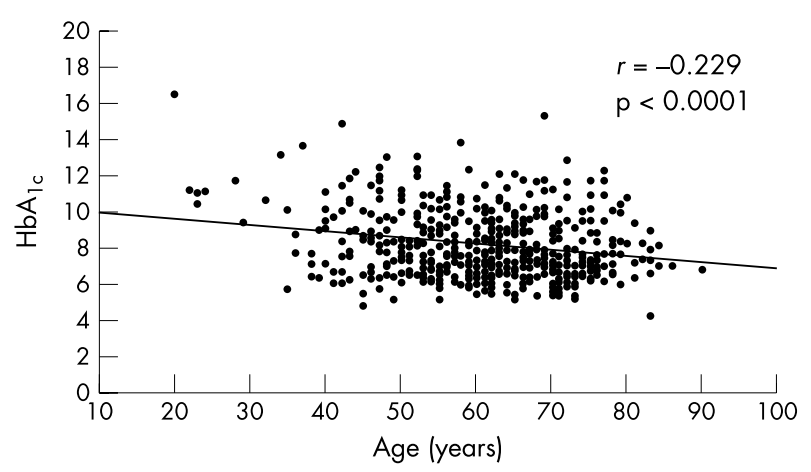

Figure 4 Relation between haemoglobin $A_{l c}$ and age in diabetic patients $(n=611)$. A significant decrease of haemoglobin $A_{1 c}$ was observed with age of diabetic patients $(p<0.0001) . r=$ correlation coefficients.

The prevalence of diabetic retinopathy in the NIDDM patients was also examined. The mean prevalence of diabetic retinopathy among the entire NIDDM population examined was $45.7 \%$. Subdividing the NIDDM population into three subgroups according to their length of diabetes $(<10$ years, $10-20$ years, and $\geqslant 20$ years) indicated that the prevalence of diabetic retinopathy significantly increased with the increased duration of diabetes $(\mathrm{p}<0.0001)$ (Fig 5$)$. For the entire population of diabetics the mean erythrocyte AR level was 10.5 (2.9) ng/mg Hb. The NIDDM population was also subdivided into four groups based on their erythrocyte AR levels with the first group possessing AR levels of less than $7 \mathrm{ng} / \mathrm{mg} \mathrm{Hb}$, the

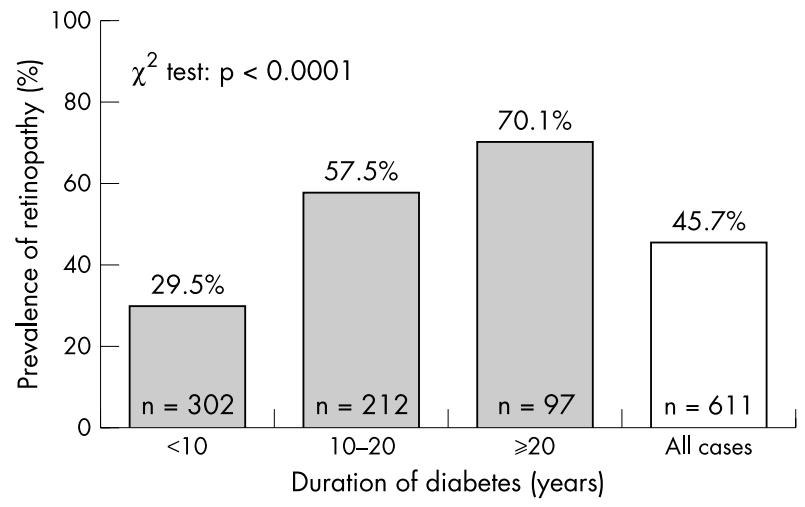

Figure 5 Relation between duration of diabetes and prevalence of retinopathy in NIDDM patients examined by $\chi^{2}$ test. The mean prevalence of diabetic retinopathy among the overall NIDDM patients was $45.7 \%$, with the prevalence of retinopathy increasing with the length of the disease $(p<0.0001)$. 


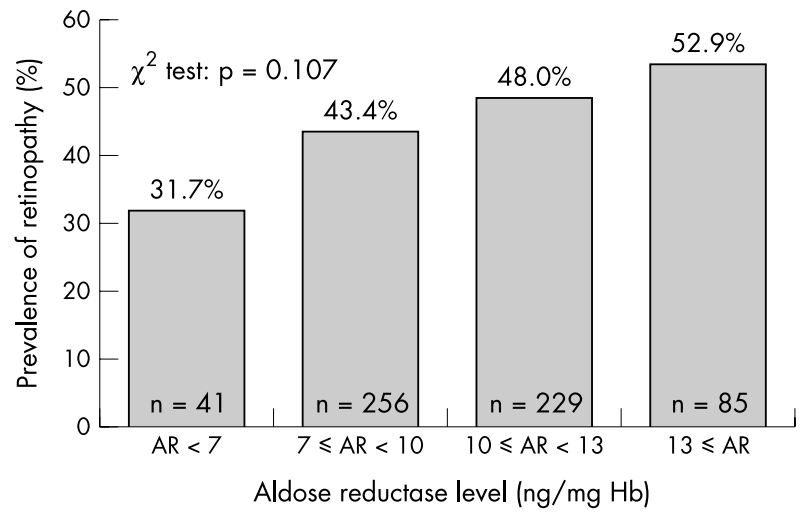

Figure 6 Relation between erythrocyte aldose reductase levels and prevalence of retinopathy in NIDDM patients divided into subgroups based on their duration of diabetes. Examined by the $\chi^{2}$ test, the prevalence of retinopathy tended to increase with increased $A R$ levels, however this correlation was not significant $(p=0.107)$.


Figure 7 Comparison between erythrocyte aldose reductase levels and prevalence of retinopathy in NIDDM patients subdivided according to their duration of diabetes. A significant correlation $(p=0.027)$ was observed in patients with a duration of less than 10 years $(A ; n=302)$. A lower correlation $(p=0.201)$ was observed in patients with $10-20$ years of diabetes $(B ; n=212)$ while the lowest correlation $(p=0.559)$ was observed in patients with diabetes for more than 20 years $(C ; n=97)$. second AR between 7 and $10 \mathrm{ng} / \mathrm{mg} \mathrm{Hb}$, the third between 10-13 ng/mg $\mathrm{Hb}$, and the fourth above $13 \mathrm{ng} / \mathrm{mg} \mathrm{Hb}$. Comparison of prevalence of retinopathy with erythrocyte AR levels in the four groups indicated that the prevalence of diabetic retinopathy increased with the levels of erythrocyte AR (Fig 6); however, this correlation was not significant for the entire NIDDM population examined $(p=0.107)$. Further dividing the four groups of erythrocyte AR levels by the duration of diabetes in these patients $(<10$ years, $10-20$ years and $\geqslant 20$ years) resulted in the comparisons illustrated in Figure 7. For patients with diabetes for less than 10 years, a significant correlation between the prevalence of retinopathy and increase in erythrocyte AR levels was observed $(p=0.027)$. There was only a tendency in the $10-20$ year group $(p=0.201)$ and a further decreased tendency in patients with diabetes for more than 20 years $(p=0.559)$.

\section{DISCUSSION}

The induction of osmotic stress from the excess intracellular accumulation of sugar alcohol (polyol) can lead to altered membrane permeability and, subsequently, to biochemical changes that result in the initiation of cellular lesions. Known as the polyol osmotic theory, Kinoshita demonstrated ${ }^{13}{ }^{14}$ that this mechanism results in the formation of diabetic cataracts that can be inhibited by the administration of aldose reductase inhibitors. Similarly, aldose reductase inhibitors prevent delays in the re-epithelialisation of corneal epithelium observed in diabetic keratopathy ${ }^{15}$ and prevent the selective destruction of retinal capillary pericytes which result in the formation of diabetic retinopathy. In the galactose fed dogs pericyte loss precedes the formation of retinal microaneurysm, haemorrhage, or exudates as well as neovascularisation. ${ }^{6716}$

The ideal method for investigating the specific relation between pericyte AR levels and diabetic retinopathy is to directly determine AR levels in the retinal capillaries. This requires enucleation of the eye followed by the isolation of the retinal capillaries by trypsin digestion and biochemical analyses. Adequate biochemical analyses of pericytes at present is technically very difficult because of the overall small size of pericytes in proportion to the size of the isolated capillaries which are primarily composed of endothelial cells. Since clearly this invasive procedure cannot be clinically utilised, we have measured erythrocyte AR levels since these levels can easily be clinically determined. Erythrocyte AR activity has been shown to correlate well with AR protein levels ${ }^{17}$ and erythrocyte AR activity in dogs has been significantly correlated with AR levels in their sciatic nerves. ${ }^{12}$

The present study of 611 NIDDM diabetics confirms that the prevalence of diabetic retinopathy significantly increases with the increased duration of diabetes and there is a tendency, though non-significant, for increased prevalence of retinopathy to correlate with increased levels of erythrocyte AR. Furthermore, subdividing the diabetics into three groups based in their duration of diabetes suggested that increased erythrocyte AR levels significantly correlated with the prevalence of retinopathy in patients diabetic for less than 10 years but that there is only a tendency for correlation with increased duration of diabetes. The decrease of $\mathrm{HbA}_{1 c}$ with the age in diabetic patients appears to be the results of long term good glucose control by the treatment of diabetics.

Similar observed increases in erythrocyte AR levels with the prevalence of diabetic complications have been reported by others. Erythrocyte AR levels have been reported to be higher in NIDDM diabetics with overt neuropathy compared to those without demonstrable neuropathy, and these increased AR levels have been identified by multivariate logistic regression analysis as an independent risk factor in addition to duration of disease. ${ }^{18}$ Similarly, erythrocyte AR levels were identified as an independent risk factor for severe diabetic neuropathy in a 
small group of NIDDM diabetics who developed severe neuropathy within 5 years of diagnosis where a $43 \%$ higher level of erythrocyte AR was observed compared to similar diabetics with no or mild neuropathy. ${ }^{19}$ Small, but significant increases in erythrocyte AR levels have also been reported in NIDDM patients with overt nephropathy compared to diabetics with no complications or microalbuminuria or only retinopathy. ${ }^{20}$ Similarly, increased erythrocyte AR levels have been reported in NIDDM patients with neuropathy ${ }^{21}$ or retinopathy ${ }^{21} 22$ compared to similar diabetics without these complications. NIDDM patients with erythrocyte AR levels over $12.0 \mathrm{ng} / \mathrm{mg} \mathrm{Hb}$ also were reported to have a fivefold increased prevalence of proliferative retinopathy compared to diabetes with erythrocyte AR levels of under $7.4 \mathrm{ng} / \mathrm{mg} \mathrm{Hb} .^{10}$

A number of studies also report that the prevalence of retinopathy and nephropathy is linked to the presence of a Z-2 allele in the aldose reductase gene. However, this association between the Z-2 AR allele and retinopathy is not observed in studies with poorly defined study populations or with increased duration of diabetes. ${ }^{20} 23$ The Z-2 allele appears to be associated with higher erythrocyte AR levels. ${ }^{11}$

The purpose of this investigation was to clarify the relation between AR level and the prevalence of diabetic retinopathy in NIDDM patients and further studies are needed for elucidate the specific relation between AR levels and the formation of diabetic complications, and also to clarify the correlation between the erythrocyte AR level and severity of retinopathy. This study indicates that the involvement of AR in diabetic retinopathy is more prominent in patients with a relatively short duration of NIDDM compared with those with longer diabetic duration. The present observation that the prevalence of retinopathy is significantly increased as the erythrocyte AR level increased is consistent with the observations of others that increased AR levels are associated with the prevalence of diabetic complications in short term diabetics. This suggests that the inhibition of AR in the early stages of diabetes may be beneficial in delaying the onset and progression of diabetic retinopathy. While this has clearly been demonstrated in dogs and rats, ${ }^{5625}{ }^{26}$ no beneficial effect was observed in the 3 year Sorbinil Retinopathy Trial Research Group. ${ }^{27}$ Here, the percentage of patients whose severity of retinopathy grade at the 41 month follow up had worsened by two or more levels was significantly different in the sorbinil (ARI) and placebo group. ${ }^{16}$ It should be noted, however, that even the strict blood sugar control gave no beneficial effects within 3 years in the DCCT clinical trial. ${ }^{1}$ This suggests that the 3 year follow up time for the Sorbinil Retinopathy Trial was too short to significantly evaluate the efficacy of an AR inhibitor. Therefore, we conclude that the inhibition of aldose reductase along with tight control of blood glucose levels at the early stages of diabetes may be beneficial in preventing pericyte degeneration in the retinal capillaries that can lead to the development and progression of diabetic retinopathy.

\section{Authors' affiliations}

N Oishi, E Kubo, Y Takamura, Y Akagi, Department of

Ophthalmology, Fukui Medical University, Fukui, Japan

K Maekawa, T Tanimoto, National Institute of Health Sciences, Osaka Branch, Osaka, Japan

Correspondence to: Yoshio Akagi, MD, PhD, Department of Ophthalmology, Fukui Medical University, Matsuoka, Yoshida-gun, Fukui, 910-1 193, Japan; akagiy@fmsrsa.fukui-med.ac.jp

Accepted for publication 19 June 2002

\section{REFERENCES}

1 The Diabetes Control and Complications Trial Research Group. The effect of intensive diabetes treatment on the progression of diabetic retinopathy in insulin-dependent diabetes mellitus. Arch Ophthalmol $1995 ; 113: 36-51$.

2 Siфlie AK, Stephenson J, Aldington S, et al the EURODIAB IDDM Complications Study Group. Retinopathy and vision loss in insulin-dependent diabetes in Europe. Ophthalmology 1997:104:252-60.

3 Akagi Y, Kador PF, Kuwabara T, et al. Aldose reductase localization in human retinal mural cells. Invest Ophthalmol Vis Sci 1983;24:1516-19.

4 Akagi Y, Yajima Y, Kador PF, et al. Localization of aldose reductase in the human eye. Diabetes 1984:33:562-6.

5 Kador PF, Akagi Y, Terubayashi $\mathrm{H}$, et al. Prevention of pericyte ghost formation in retinal capillaries of galactose-fed dogs by aldose reductase inhibitors. Arch Ophthalmol 1988;106:1099-1102.

6 Kador PF, Akagi Y, Takahashi Y, et al. Prevention of retinal vessel changes associated with diabetic retinopathy in galactose-fed dogs by aldose reductase inhibitors. Arch Ophthalmol 1990;108:1301-9.

7 Takahashi Y, Wyman M, Ferris F, et al. Diabeteslike preproliferative retinal changes in galactose-fed dogs. Arch Ophthalmol 1992;110:1295-302

8 Heesom AE, Millward A, Demaine AG. Susceptibility to diabetic neuropathy in patients with insulin dependent diabetes mellitus is associated with a polymorphism at the $5^{\prime}$ end of the aldose reductase gene. J Neurol Neurosurg Psychiatry 1998:64:213-16.

9 Kao YL, Donaghue K, Chan A, et al. A novel polymorphism in the aldose reductase gene promoter region is strongly associated with diabetic retinopathy in adolescents with type I diabetes. Diabetes 1999;48: 1338-40

10 Ogata $M$, Iwasaki $N$, Kanamori $M$, et al. Polymorphism of the aldose reductase gene is associated with aldose reductase protein levels and diabetic microangiopathy Diabetologia 1997:40:A168.

11 Ko BC, Lam KS, Wat NM, et al. An (A-C)n dinucleotide repeat polymorphic marker at the $5^{\prime}$ end of the aldose reductase gene is associated with early-onset diabetic retinopathy in NIDDM patients. Diabetes 1995;44:727-32.

12 Tanimoto T, Maekawa K, Okada S, et al. Clinical analysis of aldose reductase for differential diagnosis of the pathogenesis of diabetic complication. Anal Chim Acta 1998;365:285-92.

13 Kinoshita JH. Cataracts in galactosemia. Invest Ophthalmol 1965;4:786-99.

14 Kinoshita JH. Mechanisms initiating cataract formation. Invest Ophthalmol Vis Sci 1974;13:713-24.

15 Ohashi Y, Matsuda M, Hosotani H, et al. Aldose reductase inhibitor (CT-1 12) eyedrops for diabetic corneal epitheliopathy. Am J Ophthalmol 1988;105:233-8.

16 Kobayashi T, Kubo E, Takahashi Y, et al. Retinal vessel changes in galactose-fed dogs. Arch Ophthalmol 1998;116:785-9.

17 Nishimura C, Hamada Y, Tachikawa T, et al. Enzyme immunoassay for erythrocyte aldose reductase. Clin Chem 1994;40:889-94

18 Ito T, Nishimura C, Takahashi Y, et al. The level of erythrocyte aldose reductase: a risk factor for diabetic neuropathy? Diab Res Clin Pract 1997:36:161-7.

19 Takahashi Y, Tachikawa T, Ito T, et al. Erythrocyte aldose reductase protein: a clue to elucidate risk factors for diabetic neuropathies independent of glycemic control. Diab Res Clin Pract 1998;42:101-7.

20 Maeda S, Haneda M, Yasuda H, et al. Diabetic nephropathy is not associated with the dinucleotide repeat polymorphism upstream of the aldose reductase (ALR2) gene but with erythrocyte aldose reductase content in Japanese subjects with type 2 diabetes. Diabetes content in Japanese
1999:48:420-2.

21 Ohnishi A, Tanimoto T, Nagaki J, et al. Relationship between erythrocyte aldose reductase level and diabetic complications among 98 NIDDM patients. J Japan Diabet Soc 1996:39:783-8.

22 Nishimura C, Saito T, Ito T, et al. High levels of erythrocyte aldose reductase and diabetic retinopathy in NIDDM patients. Diabetologia 1994;37:328-30.

23 Heesom AE, Hibberd ML, Millward A, et al. Polymorphism in the 5'-end of the aldose reductase gene is strongly associated with the development of diabetic nephropathy in type I diabetes. Diabetes 1997;46:287-91.

24 Chistyakov DA, Turakulov RI, Gorashko NM, et al. Polymorphism of a dinucleotide repeat within the aldose reductase gene in normalcy and insulin-dependent diabetes with vascular complications. Molekul Biol (Moscow) 1997:31:778-83.

25 Nevenschwander H, Takahashi Y, Kador PF. Dose-dependent reduction of retinal vessel changes associated with diabetic retinopathy in galactose-fed dogs by the aldose reductase inhibitor M79175. J Ocular Pharmacol Therap 1997;13:517-28.

26 Robison WG Jr, Laver N. Ocular lesions in animal models of human diabetes. In: Shafrir E, ed. Lessons from Animal Diabetes IV 1993: 145-63.

27 Sorbinil Retinopathy Trial Research Group. A randomized trial of sorbinil, an aldose reductase inhibitor, in diabetic retinopathy. Arch Ophthalmol 1990;108:1234-44. 